\title{
A Human Case of Zoonotic Dog Tapeworm, Dipylidium caninum (Eucestoda: Dilepidiidae), in China
}

\author{
Peng Jiang, Xi Zhang, Ruo Dan Liu, Zhong Quan Wang*, Jing Cui* \\ Department of Parasitology, Medical College, Zhengzhou University, Zhengzhou 450052, China
}

\begin{abstract}
We described a human case of zoonotic dog tapeworm, Dipylidium caninum (Eucestoda: Dilepidiidae), rarely occurring in China. The mother of a 17 month-old boy noted the appearance of small white and active worms over a month period in her son's feces, but the boy was asymptomatic except mild diarrhea. We observed 3 tapeworm proglottids resembling cucumber seeds in his stool sample. Microscopically, each proglottid had 2 genital pores, 1 on each lateral edge, and numerous egg capsules in the uterus. The patient was successfully treated with a single oral dose of praziquantel. Adult worms were recovered in the diarrheic stool after praziquantel treatment and purgation. His family had household pet dogs for several years, and he might have acquired the infection by ingestion of infected fleas of his pet dogs. A history of dog or cat pets and flea bites may be important clues to diagnosis of $D$. caninum infection. The infected pets should also be treated.
\end{abstract}

Key words: Dipylidium caninum, dipylidiasis, human case, China

\section{INTRODUCTION}

Dipylidiasis is a neglected parasitic zoonosis caused by Dipylidium caninum, a common intestinal tapeworm of dogs and cats [1]. D. caninum is taxonomically located in the Dipylidium genus, Dilepidiidae family, order Cyclophyllidea, and subclass Eucestoda [2]. It is also called the double pore tapeworm (cucumber tapeworm, dog tapeworm, or flea tapeworm). D. caninum adults are relative small, averaging about $15 \mathrm{~cm}$ in length. The scolex has 4 suckers and a conical, retractile rostellum with 1 to 8 rows of small hooks. Mature proglottids are longer than their width and contain 2 sets each of male and female reproductive organs with a genital atrium on each lateral edge [3]. Gravid proglottids are barrel-like in outline and contain 2 genital pores and hundreds of egg capsules (packets) in the uterus. The adults parasitize in the small intestine of definitive hosts (dogs and cats) where gravid proglottids detach from the strobila in groups of 2 or 3 . The proglottids can creep out of the anus and migrate to the perianal region or be passed with feces. Then, the proglottids expel typical egg packets through

- Received 5 August 2016, revised 2 November 2016, accepted 7 December 2016.

*Corresponding author (wangzq@zzu.edu; cuij@zzu.edu.cn)

(c) 2017. Korean Society for Parasitology and Tropical Medicine

This is an Open Access article distributed under the terms of the Creative Commons Attribution Non-Commercial License (http://creativecommons.org/licenses/by-nc/4.0) which permits unrestricted non-commercial use, distribution, and reproduction in any

medium, provided the original work is properly cited. their bilateral genital pores, or the egg packets are released after the proglottids dehydrate and rupture. The eggs might be ingested by the larvae of dog or cat fleas (e.g. Ctenocephalides felis, Ctenocephalides canis, and others) [4]. The oncosphere hatches in the gut of the arthropod, burrows through the wall, and develops into the infective cysticercoid in the hemocoel when the flea metamorphoses into the parasitic adult stage. Humans get infection by accidental ingestion of the infected dog or cat fleas; the cysticercoid is liberated in the intestine and develops its sexual maturity within about 20 days [5].

Human infections with D. caninum are rare, but it is more likely to occur in young children who kiss or are licked by their infected pets. There have been some reports of cases with D. caninum infection or dipylidiasis worldwide, since Linnaeus first described it in 1758. To the best of our knowledge, this infection is scattered in the world, and at least 349 human cases with dipylidiasis (including the case presented in this paper) have been reported to date, according to medical literatures of human dipylidiasis from Medline, Web of Science database, and Chinese National Knowledge Infrastructure (accessed till July 17,2016$)$. These cases with dipylidiasis were distributed in at least 24 countries; more than 100 cases in Italy, 86 cases in USA, 81 cases in Japan, 30 cases in China, 18 cases in Chile, 6 cases in Poland, 5 cases in India, 3 cases in Mexico, 2 cases each in Brazil, Canada, Sri Lanka, and Uruguay, and 1 case each in Argentine, Australia, Bulgaria, Cuba, Germany, Guate- 
mala, Puerto Rico, Romania, South Africa, Spain, Turkey, and UK. More than $2 / 3$ of the reported cases were small children.

In the present study, we report a case of $D$. caninum infection in a 17 month-old boy, rarely occurring in China. The patient might have acquired the infection through games with his pet dogs. Furthermore, clinical manifestations, diagnosis, and treatment of human dipylidiasis were discussed in this study.

\section{CASE RECORD}

A 17 month-old boy came from Henan province, central China. The boy was previously healthy, and was referred to the outpatient Department of Pediatrics, the First Affiliated Hospital of Zhengzhou University with a 1-month history of excreting worms in his feces. His mother mentioned that small, white, and active worms were seen in his stool. The boy has been previously diagnosed as enterobiasis and treated with a single oral dose of albendazole $(200 \mathrm{mg}$ ) by a local physician 16 days ago, but did not recover; the worms were still seen in his feces. Although the boy was asymptomatic, the concerned mother decided to submit a fecal sample to our department for parasitological examinations.

By inquiring, this patient's family has no travel history out of Henan, but had household pet dogs for several years. A physical examination showed that the boy was normal. He had no vomiting, fever, or appetite loss except mild diarrhea. The laboratory findings only revealed slightly reduced levels of hemoglobin (115 g/L), hematocrit (37\%), mean corpuscular volume (81.8 fl), mean corpuscular hemoglobin $(25.7 \mathrm{pg})$, and mean corpuscular hemoglobin concentration $(314 \mathrm{~g} / \mathrm{L})$. His level of IgE in serum was significantly higher than normal, and was up to several-hundred points $(255.8 \mathrm{IU} / \mathrm{ml})$. The eosinophil counts $\left(0.29 \times 10^{9} / \mathrm{L}\right)$ and the others hematological profiles were normal. The stool sample was found to be yellowish on gross appearance without foul smelling. No eggs were found under light microscopy. Three small flesh-colored proglottids resembling cucumber seeds were observed in his stool. The collected proglottids had 2 genital pores, 1 on each lateral edge, and numerous egg capsules in the uterus, and

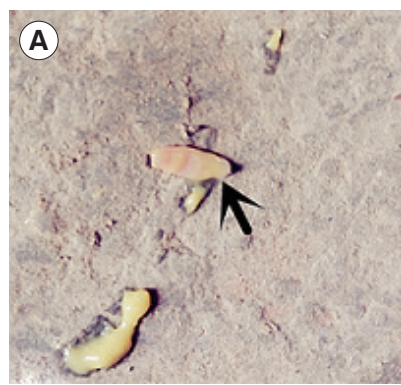

(D)

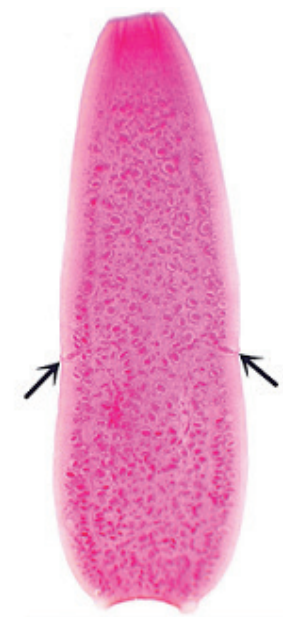

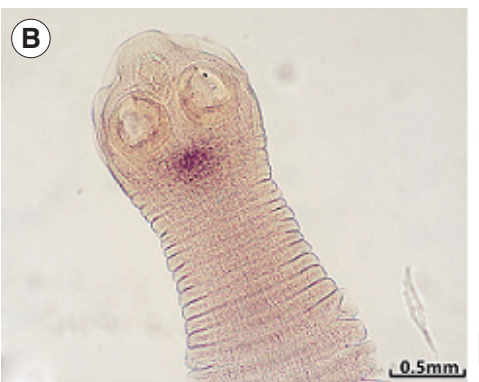

(C)

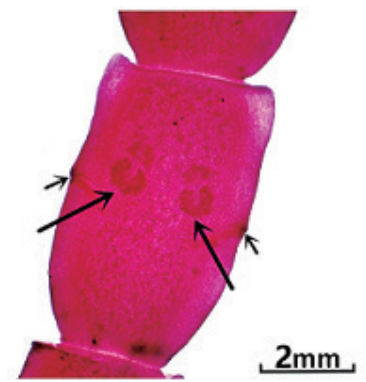

(E)
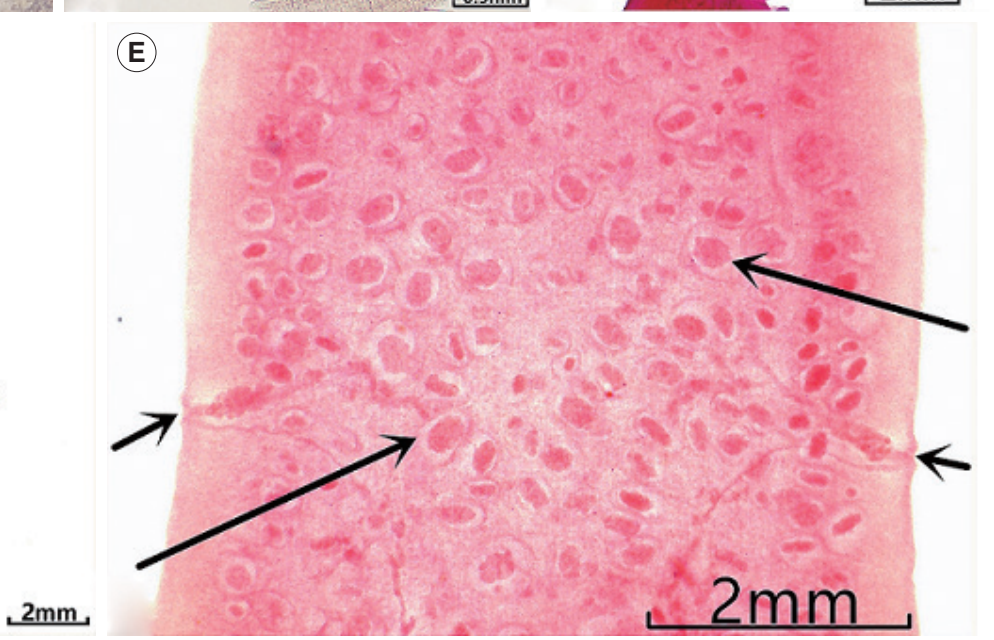

Fig. 1. Proglottids of $D$. caninum collected from the present case. (A) Actively discharged proglottid in his stool. (B) A scolex recovered in the diarrheic stool after treatment with praziquantel and purgation. (C) Mature proglottids with 2 sets of genital organs (arrow marks) (acetocarmine stained). (D) A gravid proglottid with 2 genital pores (arrows) and numerous egg capsules in the uterus. (E) Magnified view of the genital pore level of Fig. D. Genital pores (small arrows) and egg capsules (large arrows). 
they were identified as D. caninum gravid proglottids according to their morphological characteristics (Fig. 1).

The child patient was treated with a single oral dose of praziquantel ( $25 \mathrm{mg} / \mathrm{kg}$ body weight) upon the diagnosis of dipylidiasis. After $1 \mathrm{hr}$, a cup of epsom salts (10 g of magnesium sulfate in $100 \mathrm{ml}$ of warm water) was administered. From the beginning of treatment, all the fecal samples were collected and elutriated with water to detect worms for 3 days. The number of proglottids excreted on the 1st day after treatment was significantly more than those on the 2nd day, and stool examination on the 3rd day after treatment revealed no proglottids. The boy did not show any discomfort during treatment. At his follow-up at 3 months after treatment, no proglottids were observed.

\section{DISCUSSION}

The risk of $D$. caninum infection in humans is low, mainly affecting infants and young children due to their playing habits and proximity with pet dogs and cats. Among the 33 reported cases since 2000, the majority of patients, $90.9 \%$ (30/33), were children, suggesting that adult humans may be more resistant to this parasite, or children might have more chances of accidental swallowing of a flea when they kiss or are licked by their infected pets [6]. A recent study showed that D. caninum (prevalence, $72.5 \%$ ) was the most common endoparasite in both dogs and cats from Guangxi, south China [7]. Our patient's family had household pet dogs for several years, and he might have acquired the infection by ingestion of infected fleas from his pet dogs.

The patients with dipylidiasis are often asymptomatic but symptoms, such as anal pruritus, diarrhea, mild abdominal pain (usually epigastric pain), decrease in appetite, indigestion, and gastrointestinal tract disturbances, may be seen $[8,9]$. Occasionally, urticaria, eosinophilia, psychologic irritability, slight reduction in weight gain, and intestinal obstruction has also been described [10]. The statistical analysis of case reports suggested that the most common complaint is being anxious about active proglottids appearing in the perianal area, diaper, or stool. Then, the symptoms, such as anal pruritus, diarrhea, abdominal pain, and loss of appetite followed orderly in frequency. Our patient had only mild diarrhea, but his mother was anxious because of the continuous appearance of active worms in her son's feces. The clinical diagnosis of dipylidiasis usually relies on the detailed history given by the parents of the infected children.

The definite diagnosis can be made by detection of eggs or egg packets in stool. Actually, however, the egg packets can be seen only in stool samples when proglottids rupture in the intestine on rare occasions, and the eggs disintegrate rapidly. Hence, the accurate diagnosis of dipylidiasis is difficult in the absence of eggs and egg packets. Therefore, double genital pores and egg packets in the uterus of the proglottids are usually used as the diagnostic morphological characteristics of $D$. caninum. Misdiagnosis may appear when pathologists have little or no experience with this parasite. Most general practitioners and pediatricians may misdiagnose $D$. caninum infection as Enterobius vermicularis (pinworm) infection, and maggots in feces [11,12]. Our patient was misdiagnosed as enterobiasis and treated with albendazole at a local clinic. A history of dog or cat pets and flea bites may be important clues to diagnosis of $D$. caninum infection. The diagnosis was made by microscopic examinations of the excreted proglottids with characteristic 2 genital pores and egg capsules.

Various drugs have been used for treatment of human dipylidiasis, such as acranil, albendazole, atabrine (metoquine), mebendazole, niclosamide, paromomycin, praziquantel, pyrantel pamoate, and thiabendazole. However, the drugs other than praziquantel or niclosamide may not be effective in completely eliminating $D$. caninum [10]. At present, praziquantel is the drugs of choice for treatment of human dipylidiasis. $D$. caninum infection is preventable by eliminating the intermediate hosts, such as fleas, and keep people (especially children) away from companion animals like dogs and cats. Proper disposal of cat and dog feces and regular deworming of pet animals with praziquantel can help control the infection.

In conclusion, we report here a human case of D. caninum infection, rarely occurred in China. The patient was asymptomatic except for mild diarrhea, but he had a history of excreting active proglottids in his feces over 1 month. A history of dog or cat pets and flea bites may be important clues to diagnosis of $D$. caninum infection. The diagnosis can be made by recovery of excreted proglottids with characteristic 2 genital pores and egg capsules. Praziquantel is the drugs of choice for treatment of dipylidiasis. To control D. caninum infection, the government, public health officials, and medical practitioners should be aware of misdiagnosis of zoonotic dipylidiasis. Pet dogs and cats should be periodically dewormed, and their fleas should be eliminated. 


\section{CONFLICT OF INTEREST}

We have no conflict of interest related to this study.

\section{REFRENCES}

1. Narasimham MV, Panda P, Mohanty I, Sahu S, Padhi S, Dash M. Dipylidium caninum infection in a child: a rare case report. Indian J Med Microbiol 2013; 31: 82-84.

2. Cabello RR, Ruiz AC, Feregrino RR, Romero LC, Feregrino RR, Zavala JT. Dipylidium caninum infection. BMJ Case Rep 2011; doi: 10.1136/bcr.07.2011.4510.

3. John DT, Petri WA. Markell and Voge's Medical Parasitology. 9th ed. St. Louis, Missouri, USA. Elsevier Saunders. 2006, pp 274321.

4. Craig P, Ito A. Intestinal cestodes. Curr Opin Infect Dis 2007; 20: 524-532.

5. Bogitsch BJ, Carter CE, Oeltmann TN. Human Parasitology. 3rd ed. New York, USA. Academic Press. 2005, pp 278-279.
6. Roberts LS, Janovy J Jr, Gerald D. Foundations of Parasitology, 8th Ed. New York, USA. McGraw-Hill. 2009, pp 356-358.

7. Fang F, Li J, Huang T, Guillot J, Huang W. Zoonotic helminths parasites in the digestive tract of feral dogs and cats in Guangxi, China. BMC Vet Res 2015; 11: 211.

8. Ramana KV, Rao SD, Rao R, Mohanty SK, Wilson CG. Human dipylidiasis: a case report of Dipylidium caninum infection from Karimnagar. Online J Health Allied Sci 2011; 10: 28.

9. García-Agudo L, García-Martos P, Rodríguez-Iglesias M. Dipylidium caninum infection in an infant: a rare case report and literature review. Asian Pac J Trop Biomed 2014; 4 (suppl): 565-567.

10. Taylor T, Zitzmann MB. Dipylidium caninum in a 4-month old male. Clin Lab Sci 2011; 24: 212-214.

11. Weisse ME, Mullins JK, Moffett KS. A neonate with worms. Clin Infect Dis 2008; 46: 1745, 1786-1788.

12. Samkari A, Kiska DL, Riddell SW, Wilson K, Weiner LB, Domachowske JB. Dipylidium caninum mimicking recurrent Enterobius vermicularis (pinworm) infection. Clin Pediatr (Phila) 2008; 47: 397-399. 